\title{
DiVERSIDAD DE PLANTAS EXÓTICAS EN ÁREAS SOMETIDAS A DISTINTOS disturbios en el Parque Nacional Los Alerces, Chubut (ArgentinA)
}

\author{
ADRIANA KUTSCHKER ${ }^{1}$, VIVIANA HECHEM ${ }^{1}$, PATRICIA CODESAL ${ }^{2}$, MARCIA RAFAEL $^{3}$, \\ SOFÍA LÓPEZ ${ }^{4}$ y VALERIA SILVA ${ }^{4}$
}

\begin{abstract}
Summary: Diversity of exotic plants in disturbed sites in Los Alerces National Park, Chubut Province, Argentina. The proportion of exotic species in a protected area and its surrounding matrix area is influenced by the regime of natural and anthropogenic disturbances, the land use and visitors traffic to the area, enhancing susceptibility to exotic invasions. We used qualitative and quantitative approaches to compare the diversity of alien species in disturbed areas of Los Alerces National Park, northwest of the Chubut Province. We selected two sites under the disturbances: fire, grazing and camping, where transects of $50 \mathrm{~m}$ were established. In the Route $\mathrm{N}^{\circ} 71$ we used transects of $150 \mathrm{~m}$ parallel to the road. The floristic composition and the relative abundance were recorded and diversity was estimated. To detect the presence of exotic species associated with a disturbance in particular we performed a Detrended Correspondence Analysis. A total of 67 alien species were recorded, with Taraxacum officinale, Dactylis glomerata, Holcus lanatus, Rumex acetosella and Rosa rubiginosa present in all sites. Burned and camping areas presented similar species richness and diversity, while camping and grazing sites had similar floristic composition. Basic information for detection and control of exotic plants within the park, especially those considered invasive, was provided.
\end{abstract}

Key words: Conservation, disturbances, invasive species, Patagonia, protected area.

\begin{abstract}
Resumen: La proporción de especies exóticas en un área protegida y su matriz aledaña está influenciada por disturbios naturales y antrópicos, como el uso de la tierra y el tráfico de visitantes al área, que potencian la susceptibilidad a invasiones. Se analizó cuali y cuantitativamente la diversidad de especies exóticas en áreas disturbadas del Parque Nacional Los Alerces, Chubut. Se seleccionaron dos sitios sometidos a cada uno de los siguientes disturbios: incendio, pastoreo y acampe, donde se utilizaron transectas de $50 \mathrm{~m}$ de longitud. En la ruta $\mathrm{N}^{\circ} 71$ se establecieron transectas de $150 \mathrm{~m}$ paralelas al borde del camino y dispuestas cada $2 \mathrm{~km}$. Se registró la composición florística y la abundancia relativa de todas las especies exóticas presentes y se estimó la diversidad. Para comparar los sitios con distinto tipo de disturbio en base a la composición florística y a la cobertura de las diferentes especies exóticas se realizó un Análisis de Correspondencia Detendenciado. Se registraron 67 especies, con diente de león (Taraxacum officinale), pasto ovillo (Dactylis glomerata), pasto miel (Holcus lanatus), vinagrillo (Rumex acetosella) y rosa mosqueta (Rosa rubiginosa) presentes en todos los sitios. Las áreas de incendio y acampe presentaron riqueza y diversidad similar, mientras acampe y pastoreo fueron semejantes en composición especifica. Se generó información básica para la detección y control de plantas exóticas dentro del parque, especialmente de aquellas consideradas invasoras.
\end{abstract}

Palabras clave: Àrea protegida, conservación, disturbios, especies exóticas, Patagonia.

\footnotetext{
${ }^{1}$ Facultad de Ciencias Naturales. Universidad Nacional de la Patagonia S.J.B. Ruta 259, Km 16,4. Esquel (Chubut). e-mail: adrikut@gmail.com

2 Instituto de Tecnología Agropecuaria (INTA). Chacabuco 501, Esquel (Chubut).

${ }^{3}$ Secretaría Provincial de Turismo y Áreas Protegidas, Sarmiento 657, Esquel (Chubut).

${ }^{4}$ Centro de Investigación y Extensión Forestal Andino Patagónico (CIEFAP). Ruta 259, Km 16,4, Esquel (Chubut).
} 


\section{INTRODUCCIÓN}

A partir de las migraciones el hombre ha actuado como agente de dispersión de plantas y animales exóticos, fenómeno que ha aumentado a gran escala con la globalización (Mooney \& Hobbs, 2000). Por otro lado, las acciones humanas contribuyen a la ruptura de relaciones ecológicas y a la desestabilización de ecosistemas, lo que normalmente va acompañado de procesos de degradación e invasión de especies exóticas. Los principales impactos de estos procesos son la disminución y fragmentación de las poblaciones de especies nativas (Washitani, 2001), así como la modificación de la estructura y diversidad de las comunidades vegetales, cuyos efectos se extienden a la fauna que las habita (Lundgren et al., 2004; Benjamin \& Lechowicz, 2005; Alpert, 2006; Huebner \& Tobin, 2006). Si bien se sabe que aquellas plantas introducidas que se vuelven invasoras pueden modificar profundamente los ecosistemas donde se establecen, la magnitud y las características del impacto en especies nativas y ecosistemas son todavía objetos de debate (Pauchard \& Alaback, 2002, 2004).

Las invasiones biológicas han sido además reconocidas a nivel mundial como un problema potencial para la conservación de áreas protegidas debido a que causan la pérdida de diversidad y afectan la estructura de los ecosistemas y sus procesos, disminuyendo la capacidad de conservación de las mismas (Mack et al., 2000; Stohlgren et al., 2002).

A nivel global los mayores niveles de invasión de plantas exóticas en los parques nacionales corresponden a América del Norte, que alcanzan aproximadamente un 30\% (Allen et al., 2009), comparado con el $6 \%$ registrado para las reservas naturales de Europa. También hay registros de una gran cantidad de especies naturalizadas en Australasia e Islas Oceánicas, aunque la investigación en el tema es escasa (Pysek et al., 2008).

Los países de América Latina y el Caribe se han visto afectados por las invasiones biológicas de manera similar que el resto del mundo. Sin embargo, sólo en la última década la presencia de especies invasoras y sus impactos han sido considerados como un problema para la biodiversidad (Pauchard et al., 2004).
Las áreas naturales, amenazadas en forma permanente por la actividad humana (Hulme et al., 2012), deben ser protegidas en cantidad y calidad para preservar dentro de lo posible los recursos naturales y el caudal genético allí presente. Estas preocupaciones son de interés especial en las zonas templadas de Sudamérica, donde las áreas protegidas presentan altas concentraciones de especies endémicas (Ezcurra \& Puntieri, 2013).

En el caso del Parque Nacional Los Alerces, que en su mayor extensión es inaccesible al hombre (zona intangible) y sus bosques se encuentran en alto estado de pristinidad, la invasión de plantas exóticas constituye una de las principales amenazas para la conservación de su flora nativa. En su sector oriental, el bosque natural se ha ido transformando en un mosaico heterogéneo, con parches de distinta composición florística, debido a la persistencia de los impactos generados por la ganadería, el fuego y el acampe, entre otros disturbios naturales y antrópicos. Muchas de las especies introducidas por los primeros pobladores, se aclimataron perfectamente y debido a la falta de controles naturales comenzaron a dispersarse sin ayuda del hombre. Entre las especies exóticas más problemáticas del Parque Nacional Los Alerces caben mencionar: retama (Cytisus scoparius), rosa mosqueta (Rosa rubiginosa), tojo (Ulex europaeus) y coníferas exóticas (Monjeau \& Pauchet, 2006; Orellana, 2011; Ezcurra \& Puntieri, 2013).

En su doble rol de proteger la biodiversidad y permitir el acceso de visitantes al Parque para su uso público y recreación, deberían planificarse todas las acciones tendientes a mantener la estructura y el funcionamiento del ecosistema, evitando toda acción antrópica que vaya en sentido contrario. Existe un interés creciente en documentar y definir estrategias de prevención y control de las especies invasoras dentro de las áreas protegidas (Pauchard et al., 2011). En este contexto el objetivo de este estudio fue analizar distintos atributos de las comunidades de plantas exóticas en sitios sometidos a distintos tipos de disturbio, en el sector oriental del Parque Nacional Los Alerces.

\section{Materiales y Métodos}

\section{Área de estudio}

El Parque Nacional Los Alerces (PNLA) es un área protegida ubicada en la región cordillerana 


\section{A. Kutschker et al. - Plantas exóticas del Parque Nacional Los Alerces}

de la Provincia del Chubut, en el Departamento Futaleufú, sobre el límite internacional con la República de Chile (Fig. 1). Abarca una superficie de 263.000 has., de las cuales 188.000 , en el sector Oeste, corresponden a Parque Nacional y las restantes 75.000 a la Reserva Nacional (CFI, 2007). Fue creado en 1937 para proteger los bosques de lahuán o alerce (Fitzroya cupressoides), uno de los árboles de mayor longevidad del planeta, además de resguardar varias especies de fauna amenazada como el huemul (Hippocamelus bisulcus). En el área protegida están representadas la ecorregión de bosque andino-patagónico (Dominio Subantártico, Provincia Subantártica) y la altoandina (Dominio

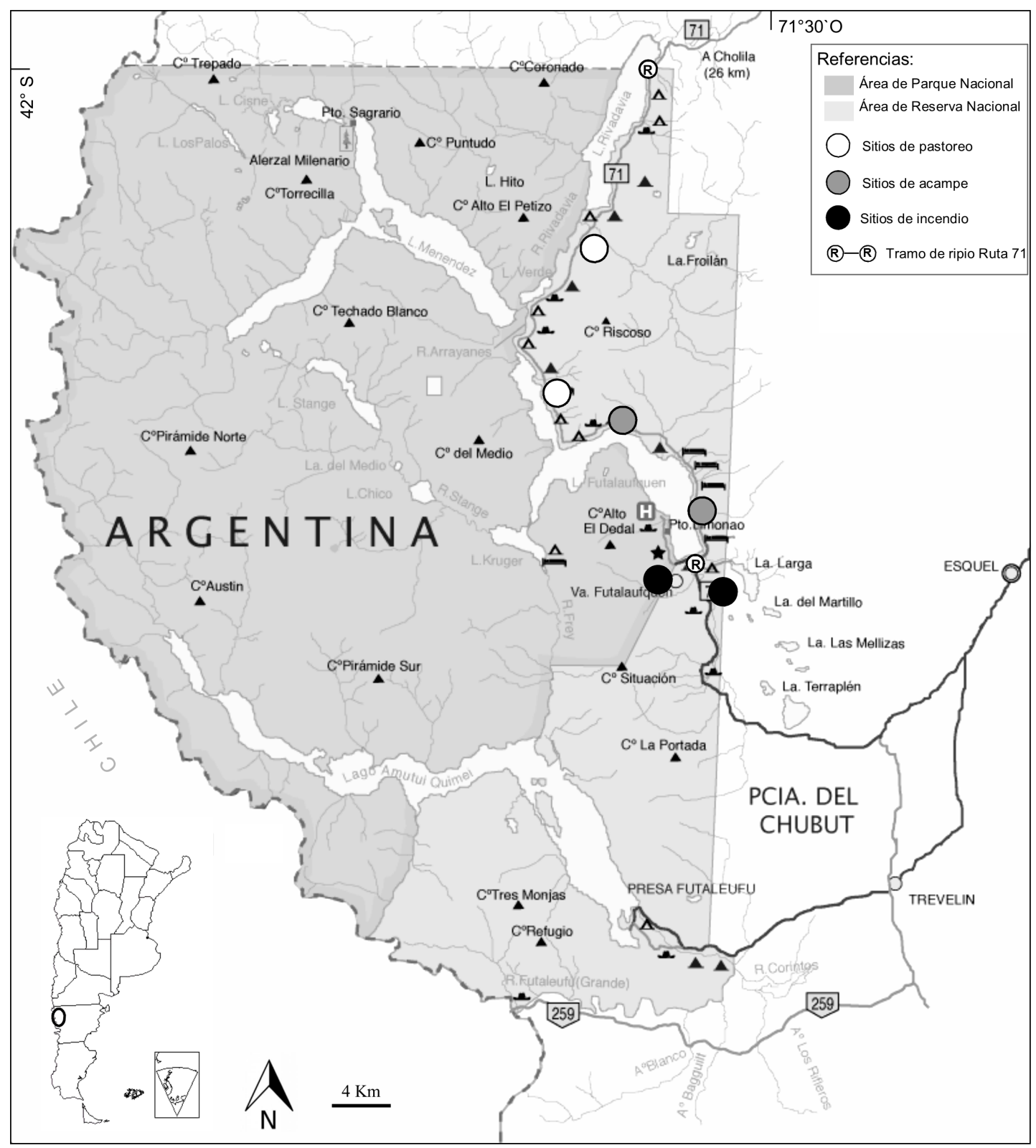

Fig. 1. Mapa del Parque Nacional Los Alerces con la ubicación de los sitios de muestreo y el tramo relevado de la Ruta $N^{\circ} 71$. 
Andino-patagónico, Provincia Altoandina) (Cabrera \& Willink, 1980). Se destacan numerosos cordones montañosos y un complejo sistema lacustre de origen glaciario, que discurre por el Parque de norte a sur (CFI, 2007).

El clima es húmedo, templado-frío y con marcada estacionalidad: los inviernos son lluviosos, con temperaturas medias de $3^{\circ} \mathrm{C}$ y nevadas intensas, los veranos son más secos, con temperaturas medias de $14^{\circ} \mathrm{C}$. Las precipitaciones disminuyen de Oeste a Este, con valores medios anuales que oscilan entre los 3000 y $4000 \mathrm{~mm}$ sobre el límite internacional, y alcanzan los 800-1000 mm en el sector oriental. Predominan vientos del cuadrante O-SO (Coronato \& Del Valle, 1988).

Este Parque presenta tres portadas de ingreso: la sur, ubicada en el sector de emplazamiento de la Presa Hidroeléctrica Futaleufú; la portada norte comunica al Parque con la localidad de Cholila y, finalmente, la portada centro, de mayor afluencia turística, y a la cual se ingresa por ruta Provincial $\mathrm{N}^{\mathrm{o}} 72$ desde Esquel $(50 \mathrm{~km})$ o Ruta Provincial N ${ }^{\mathrm{o}}$ 71 desde Trevelin $(38 \mathrm{~km})$, se continúa dentro del área protegida.

En la margen oeste del Lago Futalaufquen se encuentra la Villa de nombre homónimo, que corresponde al centro administrativo, de servicios y asentamiento de algunos pobladores. Actualmente la población del área de la Reserva está compuesta aproximadamente por 40 familias núcleo, cuyas ocupaciones en algunos casos (aprox. $25 \%$ ) se continúan en tierras ubicadas en el ejido de la ciudad de Esquel o en territorio del estado provincial. Se dedican a la cría de ganado, cultivos, extracción de leña para autoconsumo, emprendimientos extraprediales y turísticos, todas actividades que resultan un complemento para las economías domésticas (CFI, 2007).

\section{Selección de los sitios de muestreo}

Se seleccionaron dos sitios por tipo de disturbio, considerándose áreas sometidas a incendio, pastoreo y sectores de acampe, además de la ruta $\mathrm{N}^{\mathrm{o}} 71$ que conecta la Villa Futalaufquen con la portada norte. Para la elección de los sitios se trabajó en primera instancia con imágenes satelitales Landsat disponibles en la Dirección General de Bosques y Parques de la Provincia del Chubut. Estos sitios preseleccionados se definieron en terreno, teniendo en cuenta por un lado la accesibilidad y, por otro, que estuvieran sometidos a un único tipo de disturbio, para poder analizar de manera independiente el impacto de cada uno de ellos. La ubicación de cada punto de muestreo se registró con un geoposicionador satelital Garmin Etrex.

Para las áreas disturbadas por incendio, pastoreo y sitios de acampe, se seleccionaron las más uniformes posible en cuanto a escala temporal (ocurrencia del evento) y espacial, esto a los efectos de evitar variaciones debidas al tiempo transcurrido desde que se produjo el disturbio, a la superficie del área y al efecto borde.

Los sitios de acampe correspondieron a Camping Quebrada del León (Aq) (4248'41" S, 71³9’07" W), ubicado en una matriz de bosque nativo de coihue (Nothofagus dombeyi) y ciprés de la cordillera (Austrocedrus chilensis), y Camping Cascada Irigoyen (Ac) (4251'40,4' S, 71'36'20,4' W), ubicado en la margen izquierda de la Ruta $\mathrm{N}^{\mathrm{o}} 71$, camino hacia portada Norte, en un bosque dominado por coihue.

Las áreas incendiadas resultaron las más difíciles de seleccionar, ya que en general con posterioridad a un incendio los pobladores del parque utilizan los claros generados para introducir ganado. Por lo cual se eligieron dos sitios con incendios recientes, sin pastoreo. Los mismos correspondieron a Escuela $\mathrm{N}^{\mathrm{o}} 25$ (Ie) $\left(42^{\circ} 53^{\prime} 55,5^{\prime \prime} \mathrm{S}, 71^{\circ} 366^{\prime} 56,8^{\prime \prime} \mathrm{W} ; 550 \mathrm{~m}\right.$ s. m.; año del evento: 2005), ubicada en la Villa Futalaufquen en un sector dominado por coihue, maitén (Maytenus boaria) y ñire (Nothofagus antarctica), y con una superficie afectada de 15 ha. En el otro sitio denominado La Colisión (Ic) (42 $54^{\prime} 39.2^{\prime \prime} \mathrm{S}, 71^{\circ} 34^{\prime \prime} 48^{\prime \prime} \mathrm{W}$; 669 m s. m., año del evento: 2008), caracterizado por un bosque nativo de ciprés de la cordillera, maitén y ñire, el incendio se inició a $5 \mathrm{~km}$ del sector sureste del lago Futalaufquen, afectando más de 5000 ha, principalmente fuera del territorio del Parque Nacional Los Alerces.

Los sitios pastoreados fueron Potrero Bahía Rosales (Pb) (42049'09.4” S, 71'37'16.1” W; 595 m s. m.) ubicado en el extremo norte del Lago Futalaufquen, en una matriz de bosque nativo abierto dominado por coihue, ciprés de la cordillera y maitén, y Potrero Coronado (Pc) (42 $43^{\circ} 26.5^{\prime}$ " S, 71 43'39.5" W; 595 m s. m.) se encuentra en el sector de Lago Verde, en un bosque mixto de ciprés de la cordillera y maitén.

En la ruta se consideró el sector de ripio comprendido entre el cruce a la Villa Futalaufquen 


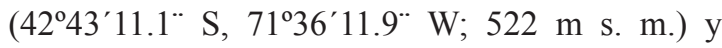
portada norte $\left(42^{\circ} 34^{\prime} 54.9^{\prime \prime} \mathrm{S}, 71^{\circ} 38^{\prime} 24.0^{\prime \prime} \mathrm{W}\right.$; $567 \mathrm{~m} \mathrm{~s} . \mathrm{m}$.), con una extensión de $55 \mathrm{~km}$. La ruta recorre el área protegida en sentido nortesur, por la margen oriental y en toda su longitud va atravesando bosques de coihue, ciprés de la cordillera, maitén y un importante sotobosque de arbustivas y herbáceas.

\section{Diseño de muestreo}

\section{Muestreo de la vegetación}

Los trabajos a campo se efectuaron en la época estival (2010 y 2011), donde las condiciones climáticas y el estado fenológico de las plantas resultaron los más favorables para su reconocimiento. Para muestrear la vegetación en los sitios sometidos a incendio, pastoreo y acampe se utilizaron transectas de $50 \mathrm{~m}$ de longitud, equidistantes $30 \mathrm{~m}$ una de otra. El tamaño de muestra (número de transectas/réplicas) se definió por sitio y en función de la superficie del área disturbada: incendio $(n=10)$, pastoreo $(n=10)$ y acampe $(n=13)$. En la ruta, debido a su extensión, se utilizó un mayor número de transectas para registrar la máxima variación posible en la composición y abundancia de especies exóticas en ambas márgenes del camino. Se trabajó con transectas de $150 \mathrm{~m}$ de longitud, ubicadas longitudinalmente y dispuestas cada $2 \mathrm{~km}$ de recorrido $(\mathrm{n}=48)$ (Matteucci \& Colma, 1980).

En todas las transectas se determinó la riqueza y la abundancia (cobertura) de las distintas especies exóticas. Para estimar la cobertura se midió la proyección de cada planta sobre una cinta métrica, incluyendo aquellos ejemplares que se encontraban a una distancia de $50 \mathrm{~cm}$ aproximadamente a cada lado de la misma. En el caso de las gramíneas la cobertura se midió de forma conjunta como Poaceae, dada la dificultad en terreno de establecer con precisión los valores individuales de cada especie. Se describió en cada sitio de muestreo la fisonomía y composición de especies de la matriz nativa circundante.

Se recolectaron ejemplares de las plantas exóticas no identificadas en terreno, material que se herborizó e identificó hasta nivel de especie. Para su determinación taxonómica se utilizó Flora Patagónica (Correa, 1969-1999) y la nomenclatura se verificó con el Catálogo de las Plantas Vasculares del Conosur (Zuloaga et al., 2008). Se clasificó a las especies según su status en: introducidas, naturalizadas e invasoras según Nuñez \& Nuñez (2007). Se incluyó además la dispersión de las especies exóticas, considerándose los siguientes mecanismos: autocoria (por la propia planta, gravedad), anemocoria (viento), hidrocoria (agua), endozoocoria (frutos consumidos por animales, dispersan las semillas al depositar las heces); epizoocoria (frutos o semillas que se adhieren a animales) (Abraham de Noir et al., 2002).

\section{Análisis de datos}

Los datos se analizaron para todos los sitios de manera conjunta, por tipo de disturbio y para cada sitio en particular. Se describió la estructura y composición florística de las comunidades de plantas exóticas. A partir de los datos de riqueza específica y abundancia relativa (para cuyo cálculo se promediaron los valores de cobertura de las distintas transectas por sitio) se calcularon los índices de diversidad de Shannon-Weaver $\left(\mathrm{H}^{\prime}\right)$, equitatividad y Dominancia (D) para cada sitio, utilizando el programa PAST versión 1.5 (Hammer et al., 2001).

Con el propósito de comparar los sitios con distinto tipo de disturbio en base a la composición florística y a los datos cuantitativos, se realizó un Análisis de Correspondencia Detendenciado (ACD). En el mismo se desestimó la inclusión de los datos de la Ruta $\mathrm{N}^{\circ} 71$, debido al mayor esfuerzo de muestreo realizado. Se utilizaron los datos de cobertura de las distintas especies exóticas por transecta, transformados como log $(\mathrm{x}+1)$, construyéndose una Matriz Básica de Datos (MBD) rectangular de $33 \times 36$, donde las muestras correspondieron a las transectas analizadas por sitio y las variables fueron las especies. Se utilizó el Programa CANOCO (Ter Braak \& Smilauer, 1998), el cual resulta útil para separar estructuras en gradientes ambientales que uno desea conocer, asumiendo una respuesta unimodal de las especies.

\section{Resultados}

Se registraron un total de 67 especies exóticas considerando todos los sitios analizados (Tabla 1), pertenecientes a 24 familias; de éstas las mejor representadas fueron Asteraceae (13 especies), Rosaceae (8) y Fabaceae (8). Del total de especies, 47 
(71\%) correspondieron a herbáceas, en tanto 11 (16\%) fueron árboles y 9 (13\%) arbustos y subarbustos. Respecto al status 56 especies son consideradas naturalizadas, e incluyen hierbas, arbustos y árboles; 8 constituyen especies invasoras, también con las tres formas de crecimiento representadas; y 3 son introducidas, dos de ellas coníferas y una gramínea. Respecto a los mecanismos de dispersión los mayores porcentajes correspondieron a autocoria $(44,8 \%)$ y anemocoria $(38,8 \%)$ (Tabla 1$)$.

La riqueza específica en las áreas sometidas a un mismo tipo de disturbio resultó superior en la ruta con 52 especies de plantas exóticas, seguida por los sitios de pastoreo con 23 especies, incendio con 21 y acampe con 20. Las especies diente de león (T. officinale), pasto ovillo (D. glomerata), pasto miel (H. lanatus), vinagrillo ( $R$. acetosella) y rosa mosqueta ( $R$. rubiginosa), estuvieron presentes en todos los sitios estudiados (Tabla 1).

Con respecto a las formas de crecimiento de las plantas exóticas que caracterizaron a cada tipo de disturbio, las herbáceas fueron las más representadas en todos los sitios y en menor proporción lo hicieron los arbustos. Los árboles se registraron en la mayoría de los sitios, con excepción de los pastoreados, en un porcentaje mayor a las especies arbustivas (Fig. 2).

El análisis por sitio reveló que las áreas con mayor riqueza de especies fueron ambas márgenes de la Ruta $\mathrm{N}^{\mathrm{o}} 71$, seguidos por pastoreo Coronado e incendio La Colisión. Los valores del índice de diversidad fueron mínimos en el área de incendio Escuela $\mathrm{N}^{\circ} 25$ y máximos en sitio de pastoreo Coronado, en tanto que para esos mismos sitios se registraron los valores máximos y mínimos de dominancia respectivamente. La equitatividad fue mayor para el área de acampe Quebrada del León, sin embargo su diversidad fue menor debido a la baja riqueza de especies (Tabla 2).

Las especies con mayor cobertura en la Ruta $\mathrm{N}^{\circ}$ 71 fueron rosa mosqueta ( $R$. rubiginosa), gramíneas, vinagrillo ( $R$. acetosella) y siete venas ( $P$. lanceolata). El pino murrayana (P. contorta var. murrayana) y retama (C. scoparius) también fueron abundantes en determinados tramos del camino. En las áreas sometidas a pastoreo dominaron las gramíneas, trébol blanco (T. repens) y lupulina (M. lupulina) en el sitio Coronado, y las especies hierba del chancho ( $H$. radicata), siete venas ( $P$. lanceolata) y gramíneas exóticas en Bahía Rosales. En el sitio incendiado Escuela $N^{\circ} 25$ dominaron retama (C. scoparius), rosa mosqueta ( $R$. rubiginosa) y

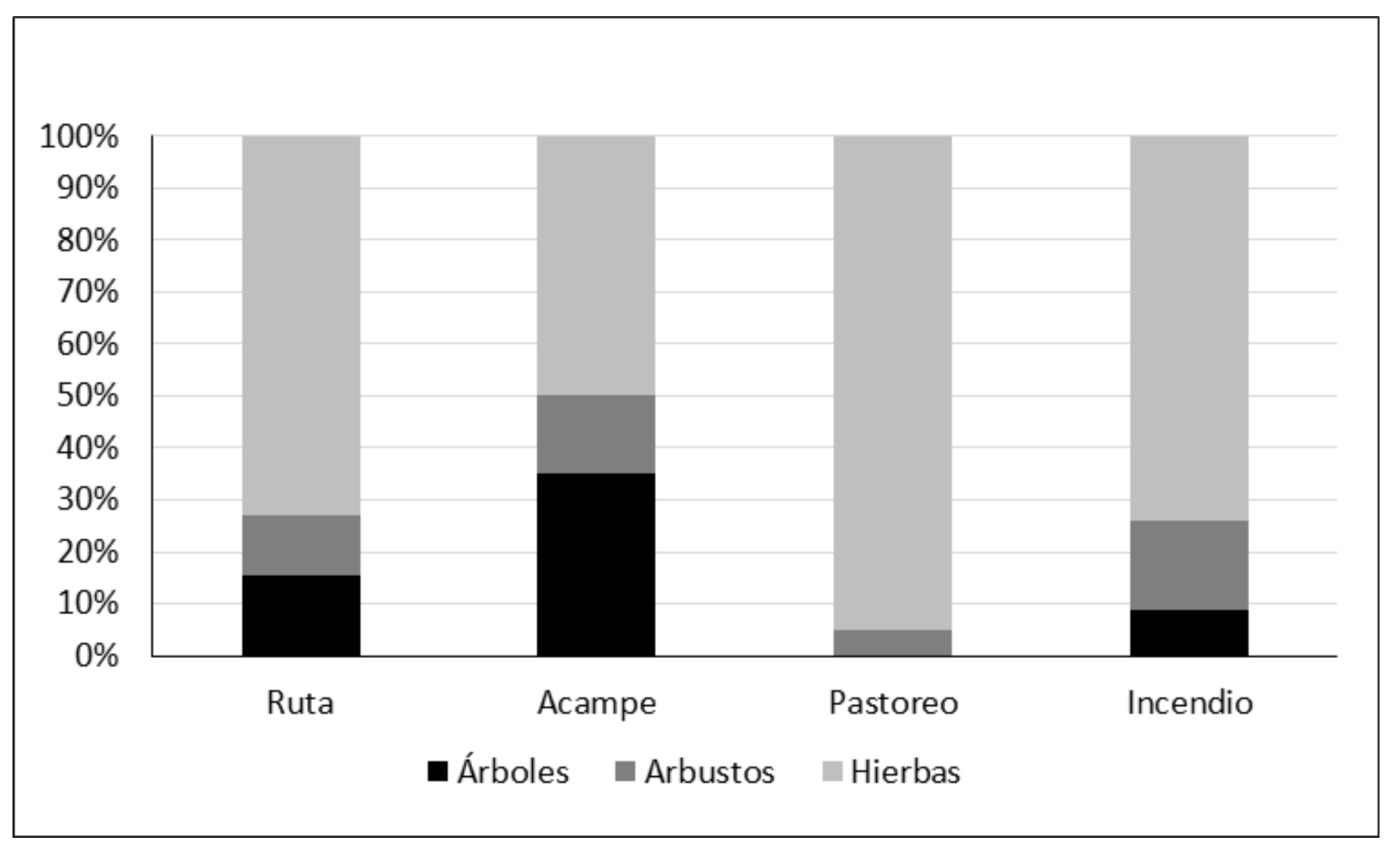

Fig. 2. Porcentaje de especies exóticas en las distintas áreas disturbadas, según su forma de crecimiento. 
Tabla 1. Especies exóticas registradas en el Parque Nacional Los Alerces, ordenadas alfabéticamente por familia. Ref.: Cód.: abreviatura de la especie utilizada en el análisis de ordenamiento. Status: Int.: introducida, Inv.: invasora, Nat.: naturalizada. Disturbio: A: acampe, I: incendio, P: pastoreo, R: Ruta № 71 Dispersión: An: anemocoria; Au: autocoria; Hi: hidrocoria; $Z_{1}$ : endozoocoria; $Z_{2}$ : epizoocoria.

\begin{tabular}{|c|c|c|c|c|c|c|}
\hline Cód. & Nombre científico & Nombre común & Hábito & Status & Disturbio & Dispersión \\
\hline & Adoxaceae & & & & & \\
\hline \multirow[t]{2}{*}{ Sam } & Sambucus nigra L. & Saúco & Árbol & Nat. & $A, I$ & $Z_{1}$ \\
\hline & Amaranthaceae & & & & & \\
\hline \multirow[t]{2}{*}{ Ama } & Amaranthus hybridus L. & Yuyo colorado & Hierba & Nat. & $P$ & $Z_{1}$ \\
\hline & Apiaceae & & & & & \\
\hline Con & Conium maculatum L. & Cicuta & Hierba & Nat. & $\mathrm{R}$ & $\mathrm{Hi}, \mathrm{Z}_{2}$ \\
\hline \multirow[t]{2}{*}{ Pas } & Pastinaca sativa L. & Chirivía & Hierba & Nat. & $\mathrm{R}$ & An \\
\hline & Asteraceae & & & & & \\
\hline Ach & Achillea millefolium $\mathrm{L}$. & Milenrama & Hierba & Nat. & $\mathrm{R}$ & $\mathrm{Au}$ \\
\hline Arc & Arctium minus (Hill) Bernh. & Bardana & Hierba & Nat. & $\mathrm{I}, \mathrm{R}$ & An, $Z_{2}$ \\
\hline Art & Artemisia absinthium L. & Ajenjo & Subarbusto & Nat. & $\mathrm{R}$ & $\mathrm{Au}$ \\
\hline Car & Carduus thoermeri Weinm. & Cardo de caballo & Hierba & Nat. & $\mathrm{I}, \mathrm{R}$ & $\mathrm{An}, \mathrm{Hi}, \mathrm{Z}_{2}$ \\
\hline Cich & Cichorium intybus L. & Achicoria & Hierba & Nat. & $\mathrm{R}$ & An \\
\hline Cir & Cirsium vulgare (Savi) Ten. & Cardo negro & Hierba & Nat. & I, P, R & An, $\mathrm{Hi}, \mathrm{Z}_{2}$ \\
\hline Cre & Crepis capillaris (L.) Wallr. & Falsa achicoria & Hierba & Nat. & $\mathrm{I}, \mathrm{P}, \mathrm{R}$ & An \\
\hline Hra & Hypochaeris radicata L. & Hierba del chancho & Hierba & Nat. & $A, P, R$ & An \\
\hline Lac & Lactuca serriola L. & Lechuga salvaje & Hierba & Nat. & $\mathrm{R}$ & $\mathrm{An}, \mathrm{Hi}$ \\
\hline Leu & Leucanthemum vulgare Lam. & Margarita & Hierba & Nat. & $\mathrm{R}$ & $\mathrm{An}, \mathrm{Au}$ \\
\hline Mat & Matricaria recutita L. & Manzanilla & Hierba & Nat. & $\mathrm{P}$ & $\mathrm{Au}$ \\
\hline Tan & Tanacetum vulgare L. & Tanaceto & Hierba & Nat. & $\mathrm{R}$ & $\mathrm{Au}$ \\
\hline \multirow[t]{2}{*}{ Tar } & $\begin{array}{l}\text { Taraxacum officinale G. Weber ex F.H. } \\
\text { Wigg. }\end{array}$ & Diente de león & Hierba & Nat. & $A, I, P, R$ & $\mathrm{An}, \mathrm{Hi}, \mathrm{Z}_{2}$ \\
\hline & Boraginaceae & & & & & \\
\hline \multirow[t]{2}{*}{ Cyn } & Cynoglossum creticum Mill. & Lengua de perro & Hierba & Nat. & $\mathrm{R}$ & $Z_{2}$ \\
\hline & Brassicaceae & & & & & \\
\hline Bni & Brassica nigra (L.) W.D.J. Koch & Pimienta negra & Hierba & Nat. & $\mathrm{P}, \mathrm{R}$ & $\mathrm{Au}$ \\
\hline Bra & Brassica rapa L. & Nabo silvestre & Hierba & Nat. & $\mathrm{R}$ & $\mathrm{Au}$ \\
\hline \multirow[t]{2}{*}{ Cap } & Capsella bursa-pastoris (L.) Medik. & Bolsa de pastor & Hierba & Nat. & $\mathrm{P}$ & An, $Z_{1}$ \\
\hline & Caryophyllaceae & & & & & \\
\hline Cer & Cerastium arvense L. & Oreja de ratón & Hierba & Nat. & $\mathrm{I}, \mathrm{R}$ & $\mathrm{Au}$ \\
\hline \multirow[t]{2}{*}{ Ste } & Stellaria media (L.) Cirillo & Capiquí & Hierba & Nat. & $P$ & $\mathrm{An}, \mathrm{Au}$ \\
\hline & Chenopodiaceae & & & & & \\
\hline \multirow[t]{2}{*}{ Che } & Chenopodium album L. & Quinoa & Hierba & Nat. & 1 & $\mathrm{Z}_{1}$ \\
\hline & Cupressaceae & & & & & \\
\hline Cup & $\begin{array}{l}\text { Cupressus macrocarpa Hartw. ex } \\
\text { Gordon }\end{array}$ & Ciprés macrocarpa & Árbol & Int. & $A, R$ & $\mathrm{An}, \mathrm{Hi}$ \\
\hline
\end{tabular}


Bol. Soc. Argent. Bot. 50 (1) 2015

\begin{tabular}{|c|c|c|c|c|c|c|}
\hline Cód. & Nombre científico & Nombre común & Hábito & Status & Disturbio & Dispersión \\
\hline \multirow[t]{2}{*}{ Jun } & Juniperus communis L. & Enebro & Arbusto & Nat. & $\mathrm{R}$ & $\mathrm{Z}_{1}$ \\
\hline & Fabaceae & & & & & \\
\hline Cyt & Cytisus scoparius (L.) Link & Retama & Arbusto & Inv. & I, R & $\mathrm{An}, \mathrm{Au}$ \\
\hline Lab & Laburnum anagyroides Medik. & Lluvia de oro & Árbol & Nat. & $\mathrm{R}$ & $\mathrm{Au}$ \\
\hline Lup & Lupinus polyphyllus Lindl. & Lupino & Hierba & $\operatorname{lnv}$. & $A, R$ & $\mathrm{An}, \mathrm{Z}_{2}$ \\
\hline Med & Medicago Iupulina L. & Lupulina & Hierba & Nat. & $\mathrm{P}, \mathrm{R}$ & $\mathrm{Au}$ \\
\hline Mel & Melilotus albus Desr. & Trébol de olor blanco & Hierba & Nat. & $\mathrm{R}$ & $\mathrm{Au}, \mathrm{Z}_{2}$ \\
\hline Tpr & Trifolium pratense L. & Trébol rojo & Hierba & Nat. & $\mathrm{P}, \mathrm{R}$ & $\mathrm{Au}$ \\
\hline Tre & Trifolium repens $\mathrm{L}$. & Trébol blanco & Hierba & Nat. & $A, P, R$ & $\mathrm{An}, \mathrm{Au}$ \\
\hline \multirow[t]{2}{*}{ Ule } & Ulex europaeus L. & Tojo & Arbusto & Inv. & $\mathrm{R}$ & $\mathrm{Au}$ \\
\hline & Geraniaceae & & & & & \\
\hline \multirow[t]{2}{*}{ Ero } & Erodium cicutarium (L.) L’Hér. ex Aiton & Alfilerillo & Hierba & Nat. & $\mathrm{R}$ & $\mathrm{Au}$ \\
\hline & Hypericaceae & & & & & \\
\hline \multirow[t]{2}{*}{ Hpe } & Hypericum perforatum $\mathrm{L}$. & Hierba de San Juan & Hierba & Nat. & $A, I, R$ & $\mathrm{Au}$ \\
\hline & Lamiaceae & & & & & \\
\hline Mar & Marrubium vulgare L. & Malvarrubia & Hierba & Nat. & $\mathrm{R}$ & $\mathrm{Au}$ \\
\hline Men & Mentha $x$ piperita $\mathrm{L}$. & Menta & Hierba & Nat. & 1 & $\mathrm{Au}$ \\
\hline Ori & Origanum vulgare L. & Orégano & Hierba & Nat. & A & $\mathrm{Au}, \mathrm{Z}_{2}$ \\
\hline \multirow[t]{2}{*}{ Pru } & Prunella vulgaris $\mathrm{L}$. & Hierba mora & Hierba & Nat. & $\mathrm{P}, \mathrm{R}$ & $\mathrm{Au}$ \\
\hline & Onagraceae & & & & & \\
\hline \multirow[t]{2}{*}{ Epi } & Epilobium brachycarpum C. Presl & Epilobio & Hierba & Nat. & $\mathrm{I}, \mathrm{R}$ & An \\
\hline & Poaceae & & & & & \\
\hline Bro & Bromus tectorum $\mathrm{L}$. & Cebada & Hierba & Int. & $\mathrm{I}, \mathrm{R}$ & An \\
\hline Dac & Dactylis glomerata L. & Pasto ovillo & Hierba & Nat. & $A, I, P, R$ & An \\
\hline Hol & Holcus lanatus L. & Pasto miel & Hierba & Nat. & $A, I, P, R$ & An \\
\hline \multirow[t]{2}{*}{ Poa } & Poa pratensis $\mathrm{L}$. & Poa de los prados & Hierba & Nat. & $\mathrm{I}, \mathrm{P}, \mathrm{R}$ & An \\
\hline & Pinaceae & & & & & \\
\hline Pic & $\begin{array}{l}\text { Pinus contorta Douglas \& Loudon var. } \\
\text { murrayana }\end{array}$ & Pino murrayana & Árbol & Inv. & $A, R$ & An \\
\hline Pir & Pinus radiata D. Don & Pino radiata & Árbol & Int. & $\mathrm{R}$ & $\mathrm{An}, \mathrm{Au}$ \\
\hline \multirow[t]{2}{*}{ Pse } & Pseudotsuga menziesii (Mirb.) Franco & Pino oregón & Árbol & Inv. & $A, R$ & $\mathrm{An}, \mathrm{Au}$ \\
\hline & Plantaginaceae & & & & & \\
\hline PII & Plantago lanceolata L. & Siete venas & Hierba & Nat. & $A, P, R$ & $\mathrm{Au}$ \\
\hline PIm & Plantago major L. & Llantén mayor & Hierba & Nat. & $\mathrm{P}, \mathrm{R}$ & $\mathrm{Au}$ \\
\hline Ves & Veronica serpyllifolia L. & Verónica & Hierba & Nat. & $P, R$ & $\mathrm{Au}$ \\
\hline
\end{tabular}


A. Kutschker et al. - Plantas exóticas del Parque Nacional Los Alerces

\begin{tabular}{|c|c|c|c|c|c|c|}
\hline Cód. & Nombre científico & Nombre común & Hábito & Status & Disturbio & Dispersión \\
\hline & Polygonaceae & & & & & \\
\hline Pol & Polygonum aviculare L. & Sanguinaria & Hierba & Nat & $\mathrm{R}$ & $\mathrm{Z}_{2}$ \\
\hline Rua & Rumex acetosella L. & Vinagrillo & Hierba & Nat. & $A, I, P, R$ & $\mathrm{An}, \mathrm{Hi}, \mathrm{Z}_{2}$ \\
\hline \multirow[t]{2}{*}{ Ruc } & Rumex crispus L. & Lengua de vaca & Hierba & Nat. & 1 & An, $\mathrm{Hi}, \mathrm{Z}_{2}$ \\
\hline & Rosaceae & & & & & \\
\hline Cra & Crataegus monogyna Jacq. & Espino & Árbol & Nat. & 1 & $\mathrm{Z}_{1}$ \\
\hline Mal & Malus sylvestris Mill. & Manzano & Árbol & Nat. & $A, R$ & $\mathrm{Au}, \mathrm{Z}_{1}$ \\
\hline Pra & Prunus avium (L.) L. & Cerezo & Árbol & Nat. & $\mathrm{R}$ & $Z_{1}$ \\
\hline Prc & Prunus cerasus L. & Guindo & Árbol & Nat. & $\mathrm{R}$ & $Z_{1}$ \\
\hline Roc & Rosa canina L. & Rosa canina & Arbusto & Inv. & $\mathrm{I}, \mathrm{R}$ & $Z_{1}$ \\
\hline Ror & Rosa rubiginosa L. & Rosa mosqueta & Arbusto & Inv. & $A, I, P, R$ & $\mathrm{Z}_{1}$ \\
\hline Rid & Rubus idaeus L. & Frambuesa & Arbusto & Nat. & $A, I$ & $\mathrm{Z}_{1}$ \\
\hline \multirow[t]{2}{*}{ Rul } & Rubus ulmifolius Schott & Murra & Arbusto & Nat. & $A, R$ & $\mathrm{Z}_{1}$ \\
\hline & Rubiaceae & & & & & \\
\hline \multirow[t]{2}{*}{ Gal } & Galium aparine L. & Pega pega & Hierba & Nat. & $\mathrm{R}$ & $Z_{2}$ \\
\hline & Salicaceae & & & & & \\
\hline Sac & Salix caprea L. & Sauce cabruno & Arbusto & Nat. & $A$ & $\mathrm{Au}$ \\
\hline \multirow[t]{2}{*}{ Saf } & Salix fragilis $\mathrm{L}$. & Mimbrote negro & Árbol & Inv. & A & $\mathrm{Au}, \mathrm{Hi}$ \\
\hline & Scrophulariaceae & & & & & \\
\hline \multirow[t]{2}{*}{ Vet } & Verbascum thapsus $\mathrm{L}$. & Tabaco de indio & Hierba & Nat. & $P, R$ & $\mathrm{Au}$ \\
\hline & Urticaceae & & & & & \\
\hline Urt & Urtica urens L. & Ortiga & Hierba & Nat. & $\mathrm{P}$ & $\mathrm{Au}$ \\
\hline
\end{tabular}

Tabla 2. Valores de riqueza especifica (S), diversidad $\left(\mathrm{H}^{\prime}\right)$, equitatividad $(\mathrm{J})$ y Dominancia (D) por sitio.

\begin{tabular}{|c|c|c|c|c|c|}
\hline Tipo de disturbio & Sitios & $\begin{array}{l}\text { Riqueza } \\
\text { específica } \\
\text { (S) }\end{array}$ & $\begin{array}{l}\text { Shannon- } \\
\text { Weaver } \\
\left(\mathrm{H}^{\prime}\right)\end{array}$ & $\begin{array}{l}\text { Dominancia } \\
\text { (D) }\end{array}$ & $\begin{array}{c}\text { Equitatividad } \\
\text { (J) }\end{array}$ \\
\hline \multirow{2}{*}{ Incendio } & La Colisión & 17 & 1,796 & 0,2202 & 0,6631 \\
\hline & Escuela $N^{\circ} 25$ & 11 & 1,213 & 0,3695 & 0,5519 \\
\hline \multirow{2}{*}{ Pastoreo } & Coronado & 19 & 1,902 & 0,1874 & 0,6714 \\
\hline & Bahía Rosales & 13 & 1,513 & 0,2772 & 0,6309 \\
\hline \multirow{2}{*}{ Acampe } & Quebrada del León & 9 & 1,246 & 0,3569 & 0,6951 \\
\hline & Cascada Irigoyen & 16 & 1,576 & 0,291 & 0,6143 \\
\hline \multirow{2}{*}{ Ruta No 71} & Margen Oeste & 28 & 1,649 & 0,3548 & 0,5123 \\
\hline & Margen Este & 28 & 1,875 & 0,276 & 0,5826 \\
\hline
\end{tabular}


gramíneas, en tanto en La Colisión lo hicieron las gramíneas, vinagrillo ( $R$. acetosella) y epilobio $(E$. brachycarpum). En el camping Quebrada del León dominaron las gramíneas, siete venas (P. lanceolata) y rosa mosqueta ( $R$. rubiginosa), al igual que en Cascada Irigoyen, donde además aparece lupino ( $L$. polyphyllus).

El Análisis de Correspondencia Detendenciado (ACD) permitió obtener el ordenamiento de las unidades muestrales por sitio (Fig. 3a) y de las especies exóticas según su participación en los mismos (Fig. 3b). Se pueden identificar nubes de puntos que corresponden a transectas ubicadas en un mismo sitio y a su vez están agrupadas según el tipo de disturbio. El ordenamiento más disperso se da con las unidades ubicadas en sitios de incendio.
Las especies que se identifican con los sitios de acampe corresponden a rosa mosqueta y rosa canina (R. rubiginosa, R. canina), lupino (L. polyphyllus), murra (R. ulmifolius), gramíneas, hierba del chancho (H. radicata), hierba mora (P. vulgaris), sietevenas $(P$. lanceolata); estas últimas tres especies caracterizan también a los sitios pastoreados, donde las especies con mayor cobertura corresponden a diente de león (T. officinale), lupulina (M. lupulina), trébol blanco ( $T$. repens), amaranto ( $A$. hybridus), pimienta negra $(B$. nigra), capiquí (S. media), verónica ( $V$. serpyllifolia) y falsa achicoria (C. capillaris). En los sitios de incendio se registran especies como hipérico (H. perforatum), retama (C. scoparius), bardana (A. minus), vinagrillo (R. acetosella), epilobio (E. brachycarpum), quinoa (Ch. album), saúco (S. nigra), entre otras.
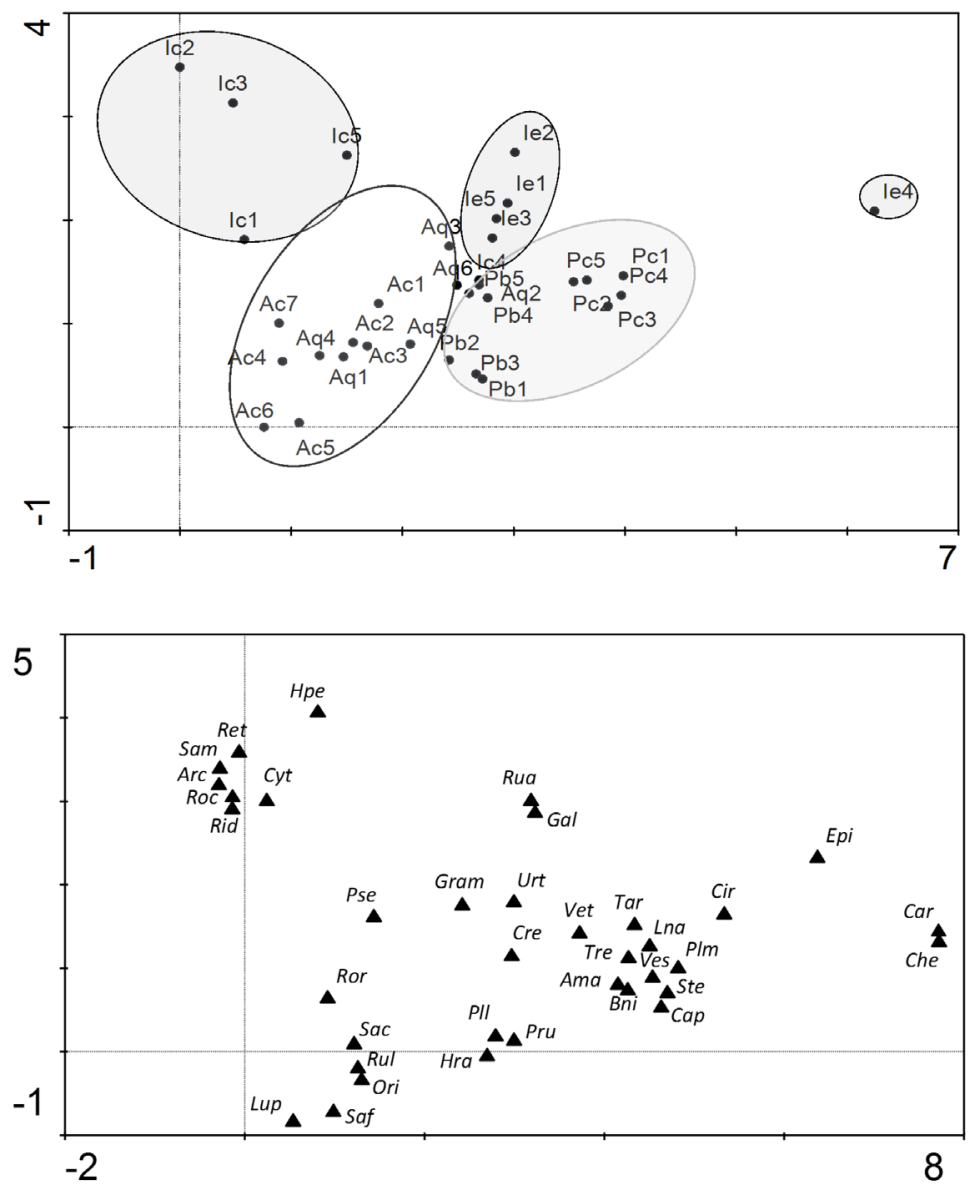

Fig. 3. Resultado del Análisis de Correspondencia Detendenciado, utilizando datos de cobertura. Se observa el ordenamiento de las unidades muestrales de cada sitio según tipo de disturbio (a) y de las especies que las caracterizan (b). 


\section{A. Kutschker et al. - Plantas exóticas del Parque Nacional Los Alerces}

\section{Discusión}

El Parque Nacional Los Alerces presenta un número importante de plantas exóticas (67) asociadas a áreas que han estado sometidas bajo distintos tipos de disturbio, algunos de los cuales perduran en la actualidad: ruta, pastoreo, incendio y acampe. Esto representa el 63\% del total de especies exóticas relevadas por Ezcurra \& Puntieri (2013) para todo el ámbito de este Parque, valores que se encuentran entre los más bajos de Patagonia Norte. Las familias Asteraceae, Rosaceae y Fabaceae incluyeron el $64 \%$ de estas especies, lo que coincide con lo descrito para áreas protegidas del centro-sur de Chile (Pauchard \& Alaback, 2004; Domínguez et al., 2006), y es similar a lo encontrado en otros continentes (Stohlgren et al., 2011).

Las hierbas representaron el $71 \%$ en general, hábito que ha sido registrado en otros estudios como el más frecuente entre las especies invasoras (Kolar \& Lodge, 2001). El análisis por sitio arrojó valores superiores al $50 \%$ en todos los casos, llegando a un $95 \%$ en los sitios pastoreados, donde además no estuvo representado el estrato arbóreo.

La Ruta $\mathrm{N}^{\mathrm{o}} 71$ fue el sitio que presentó la mayor riqueza específica, esto se debió en gran parte al mayor esfuerzo de muestreo, al recorrerse los 55 $\mathrm{km}$ de ripio que atraviesan el sector oriental del Parque hasta Portada Norte. Estudios realizados en Chile sobre especies de plantas introducidas que han invadido áreas silvestres protegidas, plantean la influencia de los bordes de caminos como vías de dispersión para las invasiones de plantas introducidas (Pauchard \& Alaback, 2004), al convertir hábitats naturales en sitios altamente vulnerables a la invasión.

A partir del análisis particular de cada sitio surgió que Pastoreo Coronado fue el que presentó la mayor riqueza específica y diversidad. Si bien el pastoreo promueve la formación de céspedes de alta diversidad florística, produce una simplificación de la estructura de la comunidad, tanto en sentido vertical como horizontal, afectando probablemente a otros niveles tróficos del ecosistema (NaiBregaglio et al., 2002). En este sitio Coronado la matriz adyacente corresponde a un bosque nativo dominado por maitén, especie de alta palatabilidad y que es consumida por el ganado vacuno, lo que dificultaría su regeneración en el área (Relva \& Veblen, 1998).
En los sitios de incendio se registraron diferencias en cuanto a la composición y abundancia de las especies. Esto puede deberse al tiempo transcurrido entre un incendio y el otro; en el caso de la Escuela $\mathrm{N}^{\circ} 25$ el incendio se produjo en el año 2005, por lo cual tuvo más tiempo de ser colonizado por especies tanto exóticas como nativas, herbáceas y arbustivas, en relación al sitio La Colisión. En este último caso el incendio ocurrió en el año 2008, y corresponde a un sector de difícil accesibilidad para el hombre, donde se registraron solamente especies herbáceas exóticas.

Las áreas de acampe fueron disímiles en cuanto a su estructura vertical. En Quebrada del León se registraron básicamente herbáceas y unos pocos ejemplares de rosa mosqueta y rosa canina en el área de camping. Corresponde a un área que fue clausurada durante un período de tiempo para su rehabilitación natural, pero que sin embargo presenta un sotobosque con un suelo muy compactado, con escasa cobertura vegetal, aún de especies exóticas. El camping Cascada Irigoyen abarca sectores con bosque nativo y claros en contacto con el lago, donde la colonización de especies exóticas ha sido mayor y se pueden encontrar ejemplares arbóreos exóticos como ciprés macrocarpa (C. macrocarpa), manzanos ( $M$. sylvestris), sauces ( $S$. sp.) y arbustos como la murra ( $R$. ulmifolius).

Todos los disturbios analizados presentan una frecuencia e intensidad que ha contribuido a la colonización de los sitios por especies exóticas, particularmente de las invasoras, modificando la estructura y composición de las comunidades vegetales nativas, como ha sido detectado en otras áreas protegidas a nivel mundial (Mack et al. 2000; Pauchard \& Alaback, 2004; Domínguez et al., 2006). Especies como vinagrillo, diente de león, pasto ovillo, pasto miel y rosa mosqueta han colonizado casi todos los hábitats modificados dentro del Parque, similar a lo encontrado en un área protegida de Chile (Domínguez et al., 2006). En particular, la rosa mosqueta presentó una alta cobertura en la mayoría de los casos. Esta especie arbustiva se caracteriza por su capacidad de reproducirse vegetativamente y por poseer frutos que representan un recurso de otoño-invierno para la fauna nativa de la región andina y en ellos, además, se privilegia la producción de semillas. Este desplazamiento temporal en la fructificación y la morfología de los frutos contribuyen al éxito en la invasión de la 
especie (Damascos, 2008; Pauchard et al., 2011).

Entre las especies ampliamente establecidas en el área protegida y consideradas peligrosas por su potencial invasor se pueden citar al lupino entre las hierbas, la retama, rosa mosqueta y tojo entre los arbustos, el pino murrayana, pino oregón y mimbrote negro, entre los árboles (Schüttler \& Santiago Karez, 2009). Otras especies con alta capacidad invasora son la rosa canina y la hierba de San Juan o hipérico, esta última se establece principalmente en los bordes de senderos y banquina de caminos o rutas (Ezcurra \& Puntieri, 2013). Todas estas especies mencionadas presentan distintos mecanismos de dispersión (anemocoria, autocoria, zoocoria), lo que confirma que ése es un aspecto más que influye en la colonización de nuevos espacios por parte de las plantas invasoras, al cual se suman la disponibilidad de propágulos de las plantas exóticas, los atributos de la comunidad nativa (riqueza de plantas y el régimen de disturbios), características de las plantas exóticas asociados con su naturalización exitosa y posterior expansión (Figueroa et al., 2004).

El conocimiento de la riqueza, abundancia y distribución de las especies exóticas aporta la información básica necesaria para diseñar actividades de prevención, detección temprana o control integrado de las especies exóticas invasoras dentro de las áreas protegidas, acciones que se están planteando como urgentes a nivel global tendientes a asegurar la conservación de la flora nativa.

\section{Agradecimientos}

A Marcelo Cabido, Estela Raffaele y un revisor anónimo por sus valiosos aportes que permitieron mejorar sustancialmente el manuscrito. Al personal y autoridades del Parque Nacional Los Alerces por su apoyo para llevar adelante investigaciones en el ámbito del área protegida. A la Secretaría de Ciencia y Técnica de la Universidad de la Patagonia San Juan Bosco por la ayuda económica para financiar el Proyecto.

\section{Bibliografía}

ABRAHAM DE NOIR, F., S. BRAVO \& R. ABDALA. 2002. Mecanismos de dispersión de algunas especies de leñosas nativas del Chaco Occidental y Serrano. Quebracho 9: 140-150.
ALLEN, J. A., BROWN, C. S. \& T. J. STOHLGREN. 2009. Non-native plant invasions of United States National Parks. Biol. Invas. 11: 2195-2207.

ALPERT, P. 2006. The advantages and disadvantages of being introduced. Biol. Invas. 8: 1523-1534.

CABRERA, A. \& A. WILLINK. 1980. Biogeografía de América Latina. Monografía $\mathrm{N}^{\circ} 13$. Secretaría General de la Organización de los Estados Americanos (OEA), Washington DC.

CONSEJO FEDERAL DE INVERSIONES (CFI). 2007. Plan Estratégico de Manejo Conjunto de las áreas este y norte del Parque Nacional Los Alerces.

CORONATO, F. R. \& H. F. DEL VALLE. 1988. Caracterización hídrica de las cuencas hidrográficas de la provincia del Chubut. Publicación Técnica, Cenpat-Conicet, Puerto Madryn.

CORREA, M. N. (dir.). 1978-1999. Flora Patagónica. Tomo VIII: parte I, III, IVa, IVb, V, y VIII. Colección científica del INTA, Buenos Aires.

DAMASCOS, M. A. 2008. La rosa mosqueta y otras rosas. CRUB-UNC, Bariloche.

DOMÍNGUEZ, E., A. ELVEBAKK, C. MARTICORENA \& A. PAUCHARD. 2006. Plantas introducidas en el Parque Nacional Torres del Paine, Chile. Gayana Bot. 63: 131-141.

EZCURRA, C. \& J. PUNTIERI. 2013. Actualización de los Planes de Manejo de los Parques Nacionales Lanín, Nahuel Huapi, Lago Puelo y Los Alerces: Flora. Informe Final Programa de mejora de la competitividad del sector Turismo, Administración de Parques Nacionales.

FIGUEROA, J. A., CASTRO, S. A., MARQUET, P. A. \& F. M. JAKSIC. 2004. Exotic plant invasions to the mediterranean region of Chile: causes, history and impacts. Revista Chil. Hist. Nat. 77: 465-483.

GELBARD, J. L. \& J. BELNAP. 2003. Roads as conduits for exotic plant invasions in a semiarid landscape. Conserv. Biol. 17: 420-432.

GENOVESI, P. \& C. SHINE. 2003. European strategy of invasive alien species. Council of Europe, Strasbourg.

HAMMER, O., D. A. T. HARPER \& P. D. RYAN. 2001. PAST: Paleontological Statistics software package for education and data analysis. Palaeontol. Electron. 4: 9.

HUEBNER, C. D. \& P. C. TOBIN. 2006. Invasibility of mature and 15 -year-old deciduous forests by exotic plants. Plant Ecol. 186: 57-68.

HULME, P. E. 2012. Weed risk assessment: a way forward or a waste of time? J. Appl. Ecol. 49: 10-19.

KOLAR, C. S. \& LODGE, D. M. 2001. Progress in invasion biology: predicting invaders. Trends Ecol. Evol. 16: 199-204. 


\section{A. Kutschker et al. - Plantas exóticas del Parque Nacional Los Alerces}

LUNDGREN, M. R., C. J. SMALL \& G. D. DREYER. 2004. Influence of Land Use and Site Characteristics on Invasive Plant Abundance in the Quinebaug Highlands of Southern New England. Northeast. Nat. 11: 313-332.

MACK, R. N., D. SIMBERLOFF, W. M. LONSDALE, H. EVANS, M. CLOUT \& F. A. BAZZAZ. 2000. Biotic invasions: causes, epidemiology, global consequences and control. Ecol. Appl. 10: 689-710.

MATTEUCCI, S. \& A. COLMA. 1982. Metodología para el estudio de la vegetación. Monografía $\mathrm{N}^{\circ} 22$. Secretaría General de la Organización de los Estados Americanos (OEA), Washington DC.

MONJEAU, J. A. \& S. PAUQUET. 2006. Estado de conservación, amenazas y prioridades de inversión en áreas protegidas andino-patagónicas. Instituto de Análisis de Recursos Naturales (IARN). Ed. Universidad Atlántida, Buenos Aires.

MOONEY, H. A. \& R. J. HOBBS. 2000. Invasive species in a changing world. Island Press, Washington DC.

NAI-BREGAGLIO, M., E. PUCHETA \& M. CABIDO. 2002. El efecto del pastoreo sobre la diversidad florística y estructural en pastizales de montaña del centro de Argentina. Revista Chil. Hist. Nat. 75: 613-623.

NUÑEZ, C. I. \& M. A. NUÑEZ. Coníferas exóticas en Patagonia: ¿Potencial invasión? Desde la Patagonia difundiendo saberes 4 (5): 2-7.

ORELLANA, I. A. 2011. Comunidades vegetales $y$ ecosistemas terrestres Parque Nacional Los Alerces. Informe Consultoría BID. Plan de Actualización del Plan de Gestión del PN Los Alerces.

PAUCHARD, A. \& P. ALABACK. 2002. La amenaza de plantas invasoras. Chile Forestal 289: 13-15.

PAUCHARD, A. \& P. ALABACK. 2004. Influence of elevation, land use, and landscape context on patterns of alien plant invasions along roadsides in protected areas of South-Central Chile. Conserv. Biol. 18: 238-248.

PAUCHARD, A., CAVIERES, L, BUSTAMANTE, R., BECERRA, P. \& E. RAPOPORT. 2004. Increasing the understanding of plant invasions in Southern South America: First symposium on Alien Plant Invasions in Chile. Biol. Invas. 6: 255-257.

PAUCHARD, A., QUIROZ, C., GARCÍA, R., ANDERSON, CH. \& M. KALIN ARROYO. 2011. Invasiones biológicas en América Latina y el Caribe: tendencias en investigación para la conservación. En: Simonetti, J. \& R. Dirzo (eds.), Conservación Biológica: Perspectivas desde América Latina, pp. 79-94. Ed. Universitaria, Santiago.
PYŠEK, P., RICHARDSON, D. M., PERGL, J., JAROŠIK, V., SIXTOVÁ, Z. \& E. WEBER. 2008 Geographical and taxonomic biases in invasion ecology. Trends Ecol. Evol. 23: 237-244.

RELVA, M. A. \& T. T. VEBLEN. 1998. Impacts of large herbivores on Austrocedrus chilensis forests in Patagonia, Argentina. Forest Ecol. Manag. 108: 27-40.

ROHLF, F. J. 2000. NTSYS-pc: numerical taxonomy and multivariate analysis system, version 2.1. Exeter Software, Setauket.

SCHÜTTLER, E. \& C. SANTIAGO KAREZ (eds.) 2009.9Especies exóticas invasoras en las Reservas de Biosfera de América Latina y el Caribe. Oficina Regional de Ciencia de la UNESCO para América Latina y el Caribe, Montevideo.

STOHLGREN, T. J., G. W. CHONG, L. D. SCHELL et al. 2002. Assessing vulnerability to invasion by non-native plant species at multiple scales. Environ. Manag. 29: 566-77.

STOHLGREN, T. J., PYŠEK, P., KARTESZ, J., NISHINO, M., PAUCHARD, A. et al. 2011. Widespread plant species: natives versus aliens in our changing world. Biol. Invas. 13: 1931-1944.

TER BRAAK, C. J. F. \& P. SMILAUER.1998. Reference manual and User's guide to Canoco for Windows: software for Canonical Community Ordination (version 4). Microcomputer power, Ithaca.

WASHITANI, I. 2001. Plant conservation ecology for management and restoration of riparian habitats of lowland Japan. Popul. Ecol. 43: 189-195.

ZULOAGA, F., O. MORRONE \& M. BELGRANO (eds.). 2008. Catálogo de las plantas vasculares del Cono Sur. Monogr. Syst. Bot. Miss. Bot. Gar. 107: 1-3348.

Recibido 30 de marzo de 2014, aceptado 20 de febrero de 2015. 
